\title{
IMPACTO SOCIO-ECONÓMICO DE LA MINERÍA EN EL CESAR, GUAJIRA Y MAGDALENA
}

\author{
Socio-economic impact of mining in Cesar, Guairá and Magdalena
}

\author{
Marlon de Jesús Correa Fernández $^{114}$
}

Fecha de recepción: 9 de abril de 2018

Fecha de aceptación: 24 de mayo de 2018

SUMARIO: 1. Introducción; 2. Metodología; 3. Impacto Socioeconómico; 4. Contextualización Histórica de la Minería en Colombia; 5. Histórico Regalías; 6. Beneficio Económico para las regiones; 7. Distribución de la Riqueza; 8. Histórico Precios del Carbón; 9. Economía Colombiana Actual; 10. Externalidades: Antecedentes en la región; 11. Violaciones a los Derechos Humanos; 12. Impacto Ambiental; 13. La consulta previa; 14. Fuerza institucional: Eficacia del control Estatal en la búsqueda de un funcionamiento óptimo entre las entidades Regionales y las Nacionales; 15. Conclusiones; 16. Bibliografía.

\footnotetext{
114 Abogado, egresado de la Universidad Sergio Arboleda seccional Santa Marta, Especialista en Derecho Probatorio y Derecho Administrativo, Magíster en Derecho de la misma Institución, Miembro del Instituto de Derecho Procesal Seccional Magdalena, Abogado Litigante y Asesor Jurídico, profesor actual en las cátedras de Derecho Probatorio en la Universidad Cooperativa de Colombia Seccional Santa Marta y Teoría General del Proceso y Procesal Civil General y Especial de la Universidad del Magdalena. Ex Defensor Público dentro del Sistema de Responsabilidad Penal de Adolescentes Regional Magdalena. Ex Defensor de Familia Regional Magdalena, Ex Abogado de la Cámara de Comercio de Santa Marta, Ex Asesor Jurídico de la Contraloría Distrital de Santa Marta, Ex Procurador Provincial de El Banco, Ex Asesor Jurídico del Hospital P. del Carmen Magdalena. Ex Profesor de la Universidad Antonio Nariño y Sergio Arboleda Santa Marta. https://orcid.org/0000-0003-3238-9914 Correo-e: marloncorreaf@hotmail.com
} 


\section{COMO SE CITA ESTE ARTíCULO (APA 6)}

Correa Fernández Marlon de Jesús (2018). Impacto Socio-económico de la Minería en el Cesar, Guajira y Magdalena. Revista Jurídica Mario Alario D’Filippo, Vol. X №. 20, pág. 132 - 153

\section{RESUMEN}

El gobierno colombiano le ha apostado a lo que llama locomotora minera, sin tener una visión sobre el impacto que tendría a futuro en las zonas donde se lleva a cabo la explotación. Por esto, el objetivo de esta investigación es determinar el impacto que ha tenido el desarrollo minero en torno al desarrollo sostenible con repercusiones económicas y sociales, en los departamentos del cesar, guajira y magdalena. Los datos presentados son de entidades del estado colombiano y ong's. En este artículo, se encontraron inconsistencias al momento de asignar licencias ambientales otorgadas por las entidades respectivas hacia algunas empresas para poder ejecutar los proyectos mineros, desconociendo anticipadamente derechos de la comunidad protegidas de estas regiones, sin utilizar la consulta previa.

\section{Palabras Claves}

Minería, externalidades, consulta previa, comunidades.

\section{ABSTRACT}

The Colombian government has bet on what it calls a mining locomotive, without having a vision about the impact it would have on the future in the areas where the exploitation takes place. For this reason, the objective of this research is to determine the impact that mining development has had on sustainable development with economic and social repercussions in the departments of Cesar, Guairá and Magdalena. The data presented are entities of the Colombian state and ngos. In this article, inconsistencies were found when assigning environmental licenses granted by the respective entities to some companies in order to execute the mining projects, previously ignoring protected community rights in these regions, without using prior consultation.

\section{Key Words}

Mining, externalities, previous consultation, communities. 


\section{INTRODUCCIÓN}

Tras la apuesta del Gobierno Nacional de elegir a la minería como motor de desarrollo económico, el país se ha beneficiado de este sector en los últimos años con las utilidades que recibió. Pero de repente las materias primas dejaron de ser rentables en el mercado internacional, como consecuencia la actividad minera generó externalidades negativas en las comunidades aledañas y un impacto ambiental muy alto a largo plazo.

En virtud de las medidas adoptadas por las autoridades ambientales, donde se le otorgan permisos a diferentes empresas para la realización de actividades de explotación minería en los departamento objeto de este estudio, sumado a la variable de la minería ilegal desempeñada en los mismos, la cual se ha querido mimetizar disfrazada de minería ancestral, viéndose perjudicada esta última, la de los pequeños mineros en comparación con la entrada a sus territorios de grandes conglomerados y empresas extranjeras con una maquinaria e infraestructura con la que no pueden competir en igualdad de condiciones.

Entorno a todos estos puntos, surge la siguiente pregunta: ¿Cuáles han sido las repercusiones que ha tenido la minería en los departamentos del Cesar, Guajira y Magdalena? Orientada, en gran medida, a encontrar una respuesta que nos permita viabilizar los herramientas que tiene el Estado Colombiano para contrarrestar los posibles efectos indeseados o proteger estimular los que son deseados, generados por la actividad minera y que repercuten de manera directa en las comunidades, especialmente la indígena y la afro descendiente.

Es por eso, que en la presentación de este artículo se quiso plasmar una estructura que evidenciara los beneficios socioeconómicos que conllevan los proyectos mineros y que se retribuyen en las regiones, haciendo especial hincapié en las que son objeto de nuestro estudio sin descuidar las del resto del país; el impacto ambiental entorno al desarrollo sostenible que conlleva la realización de estos proyectos, donde se buscará analizar si se está cumpliendo con este requisito o no.

Se analizará también, la regulación que existe respecto de esta actividad para luego entrar a sociabilizarla respecto si se está teniendo un control jurídico antes, durante y después de ser otorgada la licencia ambiental para la ejecución de los proyectos mineros, por parte de la autoridad competente.

Finalmente, se tocaré el tema de la consulta previa, la cual se vuelve de vital importancia por ser un tema que trastoca los intereses de la comunidad donde se realiza estas actividades cambiando o modificando el entorno, lo que repercute, en la necesidad del estudio sobre si se está 
cumpliendo con un desarrollo sostenible de la actividad minera arraigada en estos departamentos.

\section{Metodología}

La presente investigación se realizó siguiendo los preceptos necesarios para el desarrollo de un artículo bajo la modalidad de un artículo de revisión, con el fin mostrar los diferentes resultados obtenidos frente al tema de la minería, la cual se desarrolla en los departamentos de Cesar, Guajira y Magdalena.

Por la naturaleza de la investigación, se vio la necesidad de estructurar el artículo dándole un enfoque cualitativo, logrando así interpretar los resultados recolectados basados en el orden del método cartesiano respecto a las fuentes de diferentes posiciones en el tema. Los datos aquí presentados, hacen parte de entidades del Estado Colombiano, donde se tiene en cuenta los estudios de Fedesarrollo y Organizaciones No Gubernamentales.

El tipo de metodología que se utilizó fue el explicativo, teniendo en cuenta publicaciones primarias y secundarias donde se encontró a cabalidad estudios detallados respecto a las repercusiones de la minería entono a los departamentos que son objetos de nuestro estudio.

En este análisis, se buscó precisar y describir no solo la dificultad que presentan las comunidades, sino el medio ambiente respecto a la intervención de las empresas mineras en sus territorios en contraste con el posible crecimiento económico que esta actividad repercute en el desarrollo del país.

\section{Impacto Socioeconómico}

El Gobierno Nacional en los últimos años se mostró demasiado optimista en el movimiento de los mercados, que cada vez demandaban más productos derivados de la actividad minera, tales como: el carbón, el hierro, el oro, etc., viendo una oportunidad de atraer inversión extranjera directa (en adelante IED), para obtener utilidades que pudiesen financiar el desarrollo del país y siguiendo al mismo tiempo la tendencia de otros países para las citadas regiones en tomar parte en dicha actividad económica (Fedesarrollo, 2008, p. 11). Aunque sin quererlo, este optimismo del Gobierno Nacional tuvo consecuencias negativas para las comunidades y el medio ambiente, así las cosas:

La generación de grandes cantidades de residuos, la desaparición y contaminación de las aguas y del aire, la pérdida del suelo y de la biodiversidad, son sólo algunos de los costos ambientales que representa la minería a gran escala, de acuerdo con la investigación. (El Espectador.com, 2013, Párr. 5) 
A pesar de este conocimiento, el Estado intentó crear un plan para mitigar los efectos negativos de esta actividad económica haciendo oídos sordos a las críticas de las teorías del desarrollo sostenible como las propuestas por los economistas Samuelson \& Nordhaus (2010), sobre la explotación de recursos naturales como plan para un mejoramiento económico y social.

\section{Contextualización Histórica de la minería en Colombia}

Se puede afirmar, como bien lo dice Vicente et al, (2011) que "Antes de que este país fuese República, la actividad minera ya tenía presencia en nuestro territorio" (p. 20), y ha servido de sustento para millones de personas desde la época de la llegada de los españoles a América, e incluso esta actividad ha estado presente desde la era pre-colombina ${ }^{115}$.

No obstante, esta actividad siempre estuvo presente en el diario vivir de muchos colombianos, y fue así cuando al finalizar la década de los años noventa tras la apertura económica, que el Fondo Monetario Internacional (FMI) hizo una serie de recomendaciones para que la región Latinoamericana consiguiera su camino hacia el desarrollo económico mediante la explotación de sus recursos naturales, en el cual Colombia no fue una excepción.

El producto más fuerte al que le apostó el Gobierno Nacional fue al petróleo ${ }^{116}$, después le sigue la explotación de carbón, que la gran mayoría de producción de este producto se presenta en las minas a cielo abierto de El Cerrejón en La Guajira, Drummond en el departamento del Cesar, entre otros. Siendo este el producto que desempeña mayor impacto en la región del Caribe Colombiano.

Para el año 2006, el Gobierno Nacional estimó que el 28\% de la IED que llega a Colombia tenía como fin la minería, (Fedesarrollo, 2008, p.15), donde el producto más fuerte de la explotación industrial es el Carbón, y la región más afectada por dicha actividad es la costa norte en especial los departamentos del Cesar, La Guajira y Magdalena.

\section{Histórico Regalías}

Como se puede ver con el crecimiento de la inversión, se espera que esto también traiga beneficios al Estado Colombiano y al mismo tiempo este debe cumplir por medio de políticas fiscales un plan de redistribución de la riqueza, en especial en las regiones aledañas donde se

\footnotetext{
115 Durante la era precolombina, la minería floreció en las culturas indígenas del territorio que comprende actualmente Colombia, especialmente el periodo Preclásico (aproximadamente entre el año 1200 hasta el año 1510) fue el mayor florecimiento de la actividad minera en las denominadas Culturas Doradas como lo fueron los Muiscas, Tayronas, Zenúes, Quimbayas, etc. Siendo estas especialistas en la orfebrería y la extracción de oro en minas y riberas de los ríos con la llegada de los españoles a este territorio, mediante el esclavismo tomaron las antiguas minas de las diferentes culturas autóctonas y las explotaron en una escala industrial, haciendo que los trabajos forzados diezmaran la población de las tribus en la región.

116 El Petróleo al ser un hidrocarburo se mantendrá fuera de esta investigación pues es regido bajo una legislación distinta (Decreto 1056 de 1953)
} 
lleva a cabo la explotación. Para cumplir este objetivo, el mecanismo que utiliza el Gobierno Nacional es el sistema de regalías.

Definiremos el sistema de regalías mineras como unas "contraprestaciones económicas que se encuentran establecidas en la ley, donde los titulares (incluidos los cesionarios) tienen la obligación de pagar de manera sucesiva al Estado por la exploración y explotación de los recursos mineros". (Sunat, s.f, Párr.1)

Al pasar los años de la explotación minera, el Gobierno Nacional progresivamente ha recaudado montos mayores. Con el crecimiento de los ingresos, el Estado se permitió plantear a futuro inversiones en proyectos de desarrollo a largo plazo (especialmente en infraestructura).

"También esta tendencia de crecimiento llevó al Gobierno Nacional ser más laxo en las concesiones de los proyectos de explotación, en especial los de corto y mediano plazo". (Fedesarrollo, 2008, p.35)

Gráfico 1. Regalías mineras giradas a entidades territoriales

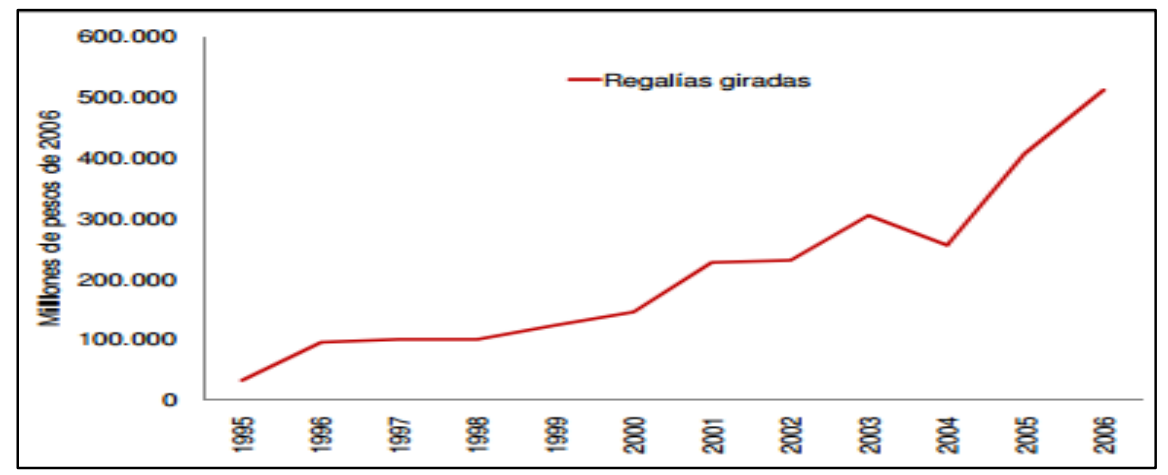

Fuente: Fedesarrollo (2008, p.34)

Como se puede apreciar en el gráfico 1, los ingresos que recibieron las entidades territoriales desde el año 1996 pasaron de ser 100.000 millones a más de 500.000 millones, esto refleja un crecimiento en el ingreso de más del $400 \%$ en un periodo de tan solo 10 años, "tiempo que generalmente toman las minas exitosas desarrollarse" (Fedesarrollo, 2008, p.16).

\section{Beneficio Económico para las regiones}

Departamento de La Guajira: Esta es la región más influenciada por la extracción de carbón de todo el territorio nacional, además este departamento, donde a partir de los años setentas, la principal actividad del departamento era el comercio con un 58\%, mientras que la minería solamente representaba un $2 \%$ de la producción departamental, pero para el año 2005 con el "boom" minero, la minería comenzó a representar el $51 \%$ de la producción departamental relegando al comercio a un 5.3\% del ingreso departamental y convirtiéndose a su vez la minería 
"boom" minero, la minería comenzó a representar el 51\% de la producción departamental, relegando al comercio a un $5.3 \%$ del ingreso departamental y convirtiéndose a su vez la minería en la principal actividad económica del departamento. (Fedesarrollo, 2008, p. 64)

Departamento del Cesar: El estudio de Fedesarrollo arrojó, que en este departamento la importancia de la minería como fuente de ingresos para la región también es muy importante, ya que "el peso de la minería en la producción total del departamento pasó de $7.3 \%$ en 1990 a un $36 \%$ en el año 2005". (Fedesarrollo, 2008, p. 68). Los cambios de la participación de la minería variaron en su producción total, principalmente en los departamentos de La Guajira y el Cesar que son los departamentos con mayor dependencia en la minería, comparado con otros departamentos (Boyacá, Córdoba y Chocó).

Distribución de la Riqueza: Como se mencionó anteriormente, el mecanismo que utiliza el Estado para distribuir los ingresos producidos por la minería se les conoce como regalías, las cuales estos son unos tributos que pide el Estado para permitir la explotación en dicha zona, a su vez estos ingresos son redistribuidos en las regiones donde la minería en este caso es la actividad principal. En los departamentos de La Guajira y el Cesar la minería es la actividad principal de ingresos. Por lo tanto, las regalías también son una parte importante de las utilidades de estos departamentos, en el estudio de 2008 de Fedesarrollo "Con un 37.56\% del total de las regalías que produce la minería nacional, las cuales van destinadas al departamento del Cesar, mientras que en el departamento de La Guajira representa un 34.15\% y para el departamento del Magdalena este porcentaje es solamente un $4.95 \%$. Con esto podemos ver que los departamentos del Cesar, La Guajira y Magdalena se benefician de un $76.66 \%$ del total de las regalías nacionales provenientes exclusivamente de la producción de carbón". (Fedesarrollo, 2008, p. 7).

\section{Histórico Precios del Carbón}

Gráfico 1. Históricos precios del carbón

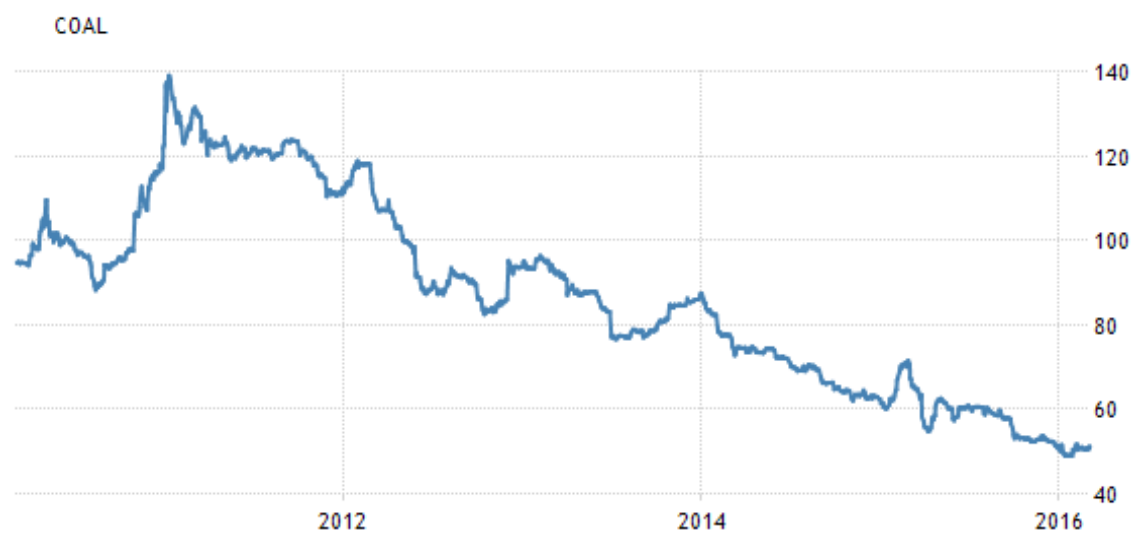

SOURCE: WWW.TRADINGECONONICS.COM I OTC

Fuente: Trading Economic (2017). 
A pesar de todo el optimismo que tuvo el Gobierno Nacional, como se puede apreciar en el gráfico 2 (tradingeconomics.com, 2016), el precio del carbón en el mercado internacional se desplomó, acabando así lo rentable del negocio de la minería y terminando con los planes del Estado, para hacer de la minería uno de los motores del desarrollo económico del país. Dejando los efectos negativos, pero ahora con unas ganancias no tan rentables para la sociedad como lo pudieron ser en el pasado.

\section{Economía Colombiana Actual}

Actualmente el Gobierno Nacional paga los errores cometidos en el pasado en confiar en la minería como motor de desarrollo a largo plazo, pues la minería actualmente no es un negocio rentable, los efectos negativos se vuelven más fuertes.

Apostarle a una inversión insostenible en el largo plazo causa aumento en el desempleo (especialmente en las regiones más dependientes de esta actividad), sumado a que las grandes compañías no están interesadas en el negocio,

...comienza a haber un crecimiento de la minería ilegal que puede ser una fuente de recursos para los grupos al margen de la ley, además de otros efectos negativos como la contaminación (ya que en la minería ilegal no hay control ambiental). (Contraloría General de la República, 2013, pp. 26 -197)

Además de todos los efectos negativos mencionados anteriormente, podemos sumar que como en una época hubo una apuesta muy fuerte solamente en la minería, esto acaba otros negocios que no son tan rentables como la minería (en el caso en que esta sea una buena opción), se dejan de producir otros productos porque todos quieren extraer minerales. Como puede ser por ejemplo "en el departamento del Cesar y sus cultivos de algodón" (ELHeraldo.com, 2013, Párr. 1). Pero estos y otros efectos negativos "podrían llevar al país a una Posible enfermedad Holandesa". (López Montaño, 2015, p. 18)

También debemos tener en cuenta el impacto ambiental que se tiene sobre las regiones y la pérdida de recursos naturales no renovables "hacen que a largo plazo el país tenga más pérdidas que ganancias" (ElHeraldo.com, 2014, Párr. 6).

\section{Externalidades: Antecedentes en la región.}

A pesar que la minería siempre existió en Colombia (incluso antes de la colonia), "la extracción a gran escala con las empresas del "boom" minero que conocemos en estos momentos se remonta a la década de los 80's" (Peace Brigades, 2011, p. 20). Las grandes concesiones a empresas extranjeras iniciaron la apertura de El Cerrejón en La Guajira. Tiempo después comenzaron 
labores en la mina de Cañaverales; Siguiendo más tarde en la década de los noventas, "comenzó la explotación en el departamento del Cesar, con las minas de Drummond en el año de 1995 en el descanso-La Loma" (Drummondco, s.f, Párr. 1) y Glencore en la Jagua Cesar en el mismo año (GLENCORE, s.f).

\section{Violaciones a los Derechos Humanos}

En las actividades de explotación minera no solo se encuentran las grandes multinacionales, "también en este negocio se pueden ver los que operan de manera ilegal, en su mayoría son grupos armados al margen de la ley" (Procuraduría General de la Nación, 2010, p. 19). Estos grupos armados generan violencia en las zonas donde operan, agudizando el conflicto, en muchos casos causando desplazamiento de la población civil. (Bauer \& al., 2011)

Con estas actividades pueden financiarse o lavar el dinero que obtienen de los cultivos ilícitos y el tráfico de estupefacientes. "En algunos casos las multinacionales han financiado a grupos ilegales para protegerse o desplazar comunidades de los lugares donde se tiene intención llevar a cabo actividades de explotación minera" (Shantz, 2011).

Se ha demostrado que demuestra que el " $87 \%$ de los desplazamientos fueron causados en municipios aledaños a explotaciones mineras y $80 \%$ de las violaciones a los Derechos Humanos en Colombia también se presentaron en zonas de municipios donde habría explotación minera industrial de multinacionales" (Fierro Morales, 2012, p. 71).

\section{Impacto Ambiental}

Los recursos naturales son uno de los elementos más afectados por la minería industrial, pero los efectos resultan ser mucho peores cuando la minería es ilegal. Como es el caso de la explotación de oro que por lo general su extracción implica la contaminación de ríos con sustancias tóxicas, tales como el cianuro o el mercurio "(un ejemplo claro es el río san salvador en el municipio de palomino en La Guajira)" (El Heraldo, 2012), afectando la flora y la fauna de los lugares por donde pasan los ríos.

También se presenta en la explotación de carbón a gran escala, por lo general esta actividad requiere la desviación del caudal de uno o más ríos para el funcionamiento de las minas, especialmente en las zonas industriales de explotación, como por ejemplo "la desviación del río Ranchería para el funcionamiento de la mina de El Cerrejón, afectando a los habitantes y la fauna de la zona" (Extractivismo en Colombia, 2015).

"La comunidad más afectada por la explotación minera en la región es sin lugar a dudas la comunidad Wayuu. Que sus tierras fueron compradas y la tribu reubicada en resguardos cerca de 
las minas" (Equipo Área de Acciones Públicas, Cajar, 2015). Como es el caso de la comunidad del municipio de Chancletas en La Guajira a tan solo 20 minutos de la mina en carro. Al vivir tan cerca de las minas las comunidades han presentado problemas respiratorios, problemas estomacales y aumento en los índices de cáncer y muerte prematura, a causa de los residuos de la mina que el viento dispersa en el ambiente.

"Según la Abogada Dora Lucy Arias, en 1982 cuando Cerrejón estaba haciendo los estudios para la explotación, estos estudios no tuvieron muy en cuenta a las comunidades indígenas de la región y añade que los procesos de consulta previa no fueron lo suficientemente transparentes y no primó del todo la buena fe" (Peace Brigades, 2011, p. 36). Los indígenas de la región no sienten suficiente respaldo del Estado y mucho menos de Cerrejón. (Bauer \& al., 2011)

\section{La consulta previa}

En el contexto del cual estamos planteando, a manera de explicación diremos que la consulta previa es un derecho fundamental y colectivo de los pueblos indígenas, donde también es un proceso de carácter público, especial y obligatorio, intercultural e interinstitucional, que debe realizarse antes de decidir, adoptar o ejecutar medidas de tipo administrativo o legislativo, programas, obras o actividades públicas o privadas que puedan afectar directamente las formas y sistemas de vida de los pueblos indígenas o su integridad étnica, cultural, espiritual, social y económica (Jimenéz García, 2016, P. 171)

Este en sí, es un derecho fundamental, pues es un reconocimiento a la autonomía, al territorio, a la diversidad étnica y al respeto por la cultura de cada etnia.

La consulta previa frente a las comunidades indígenas se encuentra en el inciso segundo del Artículo 122 del Código de Minas (Ley 685 de 2001) y está amparado por la constitución de manera implícita en los Artículos 2, 7, 44 y en el parágrafo del artículo 330 de la carta magna.

También debemos tener en cuenta que, en el ámbito internacional, la consulta previa a las comunidades indígenas actualmente se encuentra estipulada por el convenio 169 de la Organización Internacional del Trabajo (OIT) en su artículo 15:

En caso de que pertenezca al Estado la propiedad de los minerales o de los recursos del subsuelo, o tenga derechos sobre otros recursos existentes en las tierras, los gobiernos deberán establecer o mantener procedimientos con miras a consultar a los pueblos interesados, a fin de determinar si los intereses de esos pueblos serían perjudicados, y en qué medida, antes de emprender o autorizar cualquier programa de prospección o explotación de los recursos existentes en sus tierras. Los pueblos interesados deberán participar siempre que sea posible en los beneficios que 
reporten tales actividades, y percibir una indemnización equitativa por cualquier daño que puedan sufrir como resultado de esas actividades. (Convenio 189, 1989),

Este Convenio fue ratificado por el Congreso de la República en la Ley 21 del año de 1991.

Nuestra Corte Constitucional, desde muy temprano inició un desarrollo jurisprudencial con respecto al tema, a partir del año de 1997 dejó en claro el carácter fundamental de la consulta previa, y en lo que concierne a la explotación de los recursos naturales. En 2008 dicha Corte profirió las primeras declaratorias de inexequibilidad de leyes.

En el caso de la consulta previa, y en lo referente a la explotación de los recursos naturales o a la afectación de los territorios históricamente habitados por las comunidades étnicas, la Corte Constitucional ha trazado una línea jurisprudencial llegando a sentar un precedente cuando se proyectan impactos drásticos en los modos de vida y la integridad ante la autorización de medidas administrativas que tienen la potencialidad de afectar o que han afectado sus territorios, y con ello su entorno cultural.

Basándonos en el ejemplo de la Doctora Ivonne Jiménez García (2016), destacaremos las siguientes sentencias como línea jurisprudencial en el tiempo:

\begin{tabular}{cl}
\hline Providencia & \multicolumn{1}{c}{ Pronunciamiento } \\
\hline SU-039/1997 & "La Corte tuteló el derecho fundamental a la consulta previa de la comunidad indígena \\
& U'wa, debido que el Ministerio de Medio Ambiente otorgó licencia ambiental a \\
& Occidental de Colombia Inc. para actividades de explotación de hidrocarburos en áreas \\
& del resguardo de la comunidad, sin llevar a cabo un proceso previo de consulta" (Corte \\
& Constitucional, SU-039, 1997). \\
T-652/1998 & La Corte tuteló a la consulta previa del pueblo Embera del Alto Sinú como quiera que \\
& las autoridades ambientales habían otorgado licencia ambiental para la construcción \\
& de una represa en su territorio sin consultarlos previamente. (Corte Constitucional, T- \\
T-769/2009 & 652, 1998) \\
& La Corte Tuteló el derecho a la consulta previa, entre otros, de la comunidad Bachidubi, \\
& Resguardo del Río Murindó, debido a que se había concedido autorización a la \\
& Compañía Muriel Mining Corporation para la exploración y explotación de una mina de \\
& cobre, oro, y molibdeno en los departamentos de Antioquia y Chocó, proyecto Mandé \\
Norte, sin consultar con antelación a sus miembros. (Corte Constituciona, T-769, 2009) & La Corte Tuteló los derechos de la Comunidad afrocolombiana asentada en la isla de \\
T-745/2010 & Barú a la participación y a la consulta previa, debido a que la Alcaldía de Cartagena \\
celebró el contrato de concesión vial... con el Consorcio Vial Isla Barú para el estudio, \\
diseño y construcción de la vía transversal Barú y de las entradas a los puertos de los \\
poblados de la Isla, Ararca, Santana y Barú, sin llevar a cabo previamente el proceso de \\
consulta a la comunidad. Por ello se ordenó suspender la ejecución del proyecto hasta \\
que se realizara la respectiva consulta. (Corte Constitucional, T-745, 2014)
\end{tabular}




\begin{tabular}{ll}
\hline T- & La Corte Amparó el derecho a la consulta previa de la comunidad afrocolombiana \\
perteneciente al Consejo Comunitario del corregimiento La Toma, Municipio de \\
Suárez, Cauca, el cual había sido vulnerado por el otorgamiento a un particular de una \\
concesión minera para la explotación aurífera, dentro del territorio de su asentamiento \\
ancestral. (Corte Constitucional, T-1045A, 2010). \\
T-129/2011 \\
La Corte amparó los derechos a la consulta previa y a la integridad y supervivencia \\
cultural, entre otros, de la etnia Embera-Katio ubicada en los resguardos Chidima-Tolo \\
y Pescadito, departamento de Chocó, ya que varias entidades públicas como los \\
ministerios de Ambiente, Interior y Minas, Codechocó y las alcaldías de los municipios \\
como Acandí y Ungía, por acción u omisión. (Corte Constitucional, T-129, 2011) \\
La Corte amparó los derechos del pueblo indígena Achagua Piapoco, en particular su \\
derecho a la consulta previa, toda vez que fue vulnerado por los Ministerios del Interior \\
y de Justicia y de Ambiente, Vivienda y Desarrollo Territorial, la empresa Meta \\
Petroleum Limited y la Empresa Oleoducto de los Llanos Orientales -OLD-. (Corte \\
Constitucional, T-693, 2011).
\end{tabular}

La realidad en la consulta previa es que no se llega a presentar, pues muchas veces se toman acciones ilegales, "tales como el desplazamiento forzado que es un método utilizado por algunas multinacionales, por medio de grupos al margen de la ley, para llevar a cabo sus proyectos de explotación, sin tener ningún impedimento por parte de las comunidades indígenas" (Centro de Monitores de Desplazamiento Interno, 2014, pp 8-9).

Algunas entidades públicas y privadas que controlan llevan con los indígenas trámites de consulta previa, están buscando de forma incorrecta que dichos procesos sean avalados mediante el mecanismo del Fast-Track, es así que se pudo encontrar en diálogos realizados con la Defensoría del Pueblo (2017) lo siguiente:

Los pueblos indígenas, durante la reunión, solicitaron que a través de la Defensoría del Pueblo se pueda puntualizar que la reglamentación sobre el derecho fundamental a la consulta previa no es desarrollo de los acuerdos de paz y por lo tanto no debe ser objeto de este procedimiento bajo el mecanismo de Fast Track. Los representantes reiteraron que la consulta previa es un derecho humano fundamental, establecido por el Convenio 169 de la OIT de 1991 y ratificado en Colombia por la Ley 21 de 1991, por lo que sería un proceso de consulta ordinario. (Párr. 3)

Procedimiento que sin duda laguna fue utilizado para los procesos de paz con las FARC. Los líderes indígenas han demostrado consternación, pues este mecanismo no tiene nada que ver con el proceso de consulta previa y se les está vulnerando su derecho a un debido proceso, desconociendo con ello Derechos Fundamentales.

Debido a la bonanza de carbón en el departamento de La Guajira, "la mina del Cerrejón busca ser el líder del sector minero energético en la región" (Cerrejon, 2010, p. 12). Como ejemplo vemos que existen dos empresas trasnacionales que tienen sus intereses en la región. 
Entre esas está la firma Canadiense Pacific Coal que compró una mina en el municipio de Barrancas y actualmente hace uso de ella (Portafolio, 2015, Párr. 6) También la empresa Brasileña MPX está llevando obras en esta región del país como nueva inversora.

Tal como lo explica la Ley 21 (1991), se establece que los ingresos de las nuevas empresas y el crecimiento de proyectos mineros deben ser consultados por las comunidades que viven en la zona, requisito que no cumplieron las dos empresas anteriormente mencionadas.

A pesar, de todo no se ha presentado una denuncia formal, ni una tutela para este caso en específico, lo cual se considera un acto de impunidad y desidia por parte de las entidades encargadas.

Actualmente, la entidad Regional ambiental de La Guajira (CORPOGUAJIRA), maneja datos desactualizados sobre el impacto ambiental y la contaminación por parte de las industrias mineras, ya que desde 2010 no se ha presentado nuevos estudios del tema (Corpoguajira, 2010), teniendo en cuenta las nuevas solicitudes de explotación minera en la región.

Las comunidades viven un gran obstáculo frente a la necesidad de abastecimiento de agua para su consumo y para proyectos productivos. Según lo señalado en el documento de Modificación del Plan de Manejo Ambiental Integral por el Proyecto P40 elaborado por la empresa Ingeniería y Diseño - Ingetec S.A., entre los años 2006 y 2012 el Cerrejón consumió 1053,10 litros por segundo ( $\mathrm{l} / \mathrm{sg}$ ) de agua, correspondientes a concesiones otorgadas por Corpoguajira de fuentes superficiales (río Ranchería, Arroyo Bruno y Tabaco). (Cinep, 2016, p. 34)

La primera sentencia importante sobre la consulta previa en la Sierra Nevada de Santa Marta emitida por la Corte Constitucional fue la T-547 (2010), donde se explica que la Corte revisó los fallos de instancia dictados dentro de la acción de tutela interpuesta por pueblos Kogui, Arhuaco, Kankuamo y Wiwa de la Sierra Nevada de Santa Marta contra los Ministerios de Interior y Ambiente y otras Autoridades, con ocasión del inicio de las obras de Puerto Brisa. La Corte precisó que los actos administrativos que preceden el desarrollo de un proyecto de infraestructura -en ese caso portuaria-, como la respectiva licencia ambiental, así como la ejecución misma del proyecto, deben ser consultados previamente a las comunidades étnicas, no solamente cuando el proyecto se ubica dentro de los resguardos de las comunidades, sino también cuando se planea realizarlos en territorios de usos ancestrales y donde las comunidades desarrollan prácticas tradicionales.

Por otro lado tenemos los Resultados de la Sentencia T-819 (Corte Constitucional, 2014) en el Caso Sierra Nevada de Santa Marta: en esta sentencia se encontró lo siguiente: 
El Estado desconoce el convenio 169 de la Organización Internacional del Trabajo, el cual contempla el derecho de las comunidades indígenas y tribales de participar en la toma de decisiones, que afecten sus territorios, prerrogativa que además, contribuye a la protección del patrimonio material e inmaterial del país. (Corte Constitucional, 2014)

En la Sierra Nevada de Santa Marta la primera vez que el gobierno de Colombia decidió de forma legal proteger este territorio "fue en el año de 1973 mediante la Resolución número 2, emitida por el Ministerio de Gobierno esta fue modificada por la resolución 837 de Agosto de 1995 por el ministerio del interior" (Minambiente, s.f.), se delimitó todo el territorio ancestral habitado por los pueblos indígenas de toda la zona. En dicha resolución esta delimitación pasó a llamarse la línea negra que comprende 1'751.601 hectáreas cuadradas entre los departamentos de la Guajira, Magdalena y Cesar el cual será presentado en la Imagen 2. (Confetayrona, 2017).

\section{Gráfico 2. Línea negra}

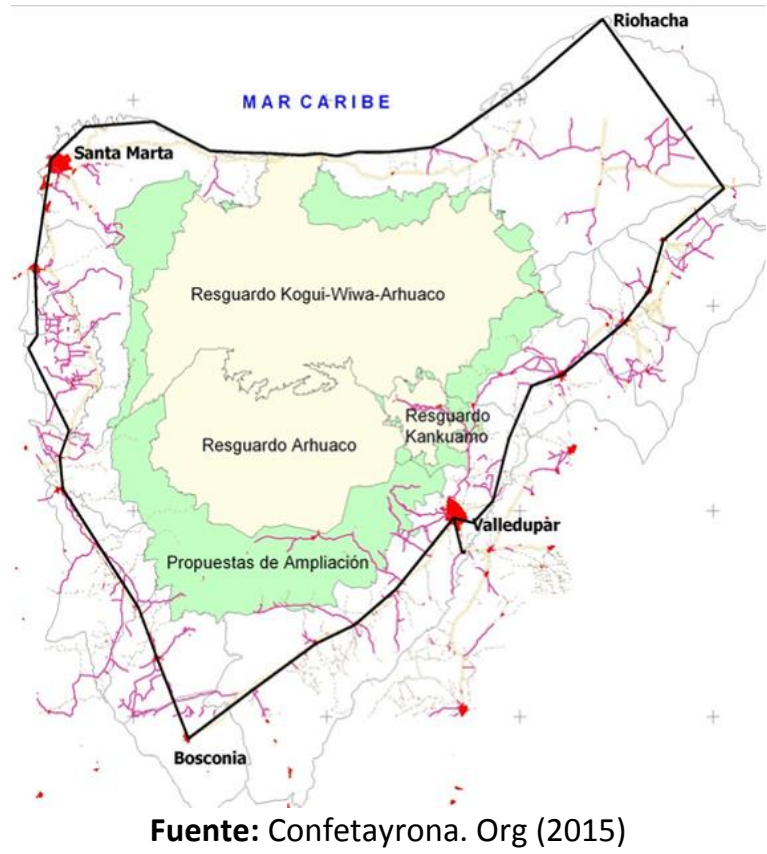

En el año 2010, La corporación Autónoma Regional del Cesar (en adelante la nombraremos con las siglas CORPOCESAR) con las resoluciones 1646 del 2010 y 1860 del 2011 concedió títulos mineros sobre la zona conocida como la línea negra, sin los permisos de las comunidades indígenas de la zona (en este caso la comunidad Arhuaca).

Hay que tener en cuenta que el Ministerio del Interior no tuvo el cuidado suficiente para determinar si CORPOCESAR estaba incurriendo en una falta, pues con el solo recibimiento de un comunicado por parte de esta entidad, el Ministerio dedujo que no había un resguardo en el lugar de la explotación, por lo tanto esta fue causal suficiente para aceptar la concesión sin una investigación pertinente. 
La falta de consulta previa en este caso específico fue bastante dañino, pues CORPOCESAR como Entidad Regional de manera irregular buscó omitir a la ley excluyendo a las comunidades indígenas de los derechos fundamentales como debido proceso y de decidir con libertad el destino de sus territorios ancestrales.

De buena forma, la Corte Constitucional dio una prelación a los derechos fundamentales como la consulta popular sobre territorios indígenas de la Sierra Nevada de Santa Marta a la comunidad Arhuaca sobre los intereses privados y la mala concepción de entidades regionales.

Lastimosamente, los indígenas de la Sierra Nevada de Santa Marta en la parte del César y La Guajira, no se sienten completamente seguros por parte del estado, pues sienten que la consulta previa como práctica no les funciona, ya que al pactar con las empresas mineras estas no cumplen con su parte. "Por ello han decidido suspender temporalmente la consulta previa en su territorio" (ELHeraldo.com, 2016, Párr. 1).

Como resultado, mientras son escritas estas líneas, los Indígenas Kankuamos y Wiwas de esta región han recibido actos vandálicos en sus Kankurwas (Casas tradicionales de su territorio ancestral), "en el municipio de Dibulla (La Guajira)" (ElEspectador.com, 2017, Párr. 4) y por otro lado el corregimiento de Guatapurí (Cesar) (ElEspectador.com, 2017, Párr. 2), llevando como resultado que reciben ataques de la minería ilegal y por la suspensión a la consulta previa.

\section{Fuerza institucional: Eficacia del control Estatal en la búsqueda de un funcionamiento óptimo entre las entidades Regionales y las Nacionales.}

"Se necesita un mayor control por parte del Estado en las entidades Regionales de Concesión Minera" (Britto Agudelo, Buitrago Suescún, Puerto Suárez, \& Ramírez, 2012, p. 15), pues en muchos casos van en contra de la misma ley, cometiendo omisiones graves para el cuidado de la Sierra Nevada de Santa Marta y sus lugares aledaños "donde a través del tiempo el Gobierno Colombiano ha tenido casi una nula fuerza institucional” (Villaraga Sanrmiento , 2008, p. 60)

Por consiguiente "Debemos tener en cuenta hasta donde es funcional la Autonomía de las Regiones en la actividad minera, pues hay que reconocer que la corrupción es abundante cuando hay debilidad Estatal” (Semana.com, 2017, Párr 2).

Tampoco debemos pensar que este problema se solucionaría si se erradicaran las entidades Regionales y se da a una Autoridad de Licencia Nacional las funciones de las entidades regionales, pues como se ha demostrado anteriormente en esta investigación, los últimos Gobiernos le apostaron a la minería como motor económico, donde aprobarían títulos mineros de manera indiscriminada, sin tener en cuenta cuales son las necesidades reales de cada región en Colombia. 
Tal es el punto que la Agencia Nacional de Minería (en adelante ANM) decidió Acelerar los trámites de solicitudes de títulos mineros para que no demoren más de 180 días. Todo para "mejorar el servicio al cliente", agilizando estas gestiones por internet (Portafolio, 2016). Teniendo en cuenta que este tipo de solicitudes requieren una investigación directa de campo, como lo son: visitar el posible lugar de exploración y explotación para determinar si existen comunidades en la zona, además del costo ambiental.

Otro inconveniente para contextualizar, es que en estos momentos hay 64 resguardos que desde hace muchos años esperan el reconocimiento legal en la Costa Atlántica, pero cuando vemos que las solicitudes de títulos mineros llegan a concederse en cuestión de meses, vemos como el Estado colombiano demuestra de manera implícita mayor prelación a las empresas mineras sobre la autonomía de las regiones para proteger las comunidades en las zonas de explotación. (Pindamó, 2008).

\section{CONCLUSIONES}

Se evidenció a lo largo de este artículo, que el Gobierno Nacional le apostó al desarrollo a largo plazo del país basado en la explotación de materias primas, que es un mercado donde los precios son demasiado volátiles para que las decisiones a largo plazo dependen de este mercado, "además de todas las externalidades negativas que trae la minería a las zonas de explotación" (Contraloría General de la República, 2013).

Para sumar a lo anterior, también contribuyó el afán de conseguir inversión extranjera sin tener en cuenta las comunidades afectadas, este error fue cometido especialmente por el Gobierno Nacional, "que no tuvo en cuenta a las entidades regionales en el proceso de dar concesiones mineras" (Salinas Alvarado, 2011, Párr. 22). Y cuando el negocio dejó de ser rentable, solo quedó la destrucción de un alivio a corto plazo.

Vemos que la consulta previa es una obligación por parte de las empresas con los resguardos indígenas que se encuentran en el lugar de exploración y explotación de este. Pero lastimosamente, ya sea por negligencia o por dolo estas no presentan la consulta a dichos resguardos.

También muchas de las licencias se dan por parte de entidades importantes como las alcaldías y los ministerios sin tener en cuenta a las comunidades, "Muchas de las entidades Ambientales, tanto nacionales como regionales, parecen desconocer (o pretender desconocer) las reglas nacionales sobre la consulta previa, muchas de las comunidades indígenas también parecen tener este desconocimiento en los procesos de consulta previa" (Corte Constitucional, 2017), haciendo 
menos sostenible el sistema minero Colombiano, llevando a muchas culturas protegidas por la constitución al riesgo de perder sus ecosistema ancestral.

\section{REFERENCIAS BIBLIOGRÁFICAS}

BRITTO Agudelo, R. A., Buitrago Suescún, Ó. Y., Puerto Suárez, J. D., \& Ramírez, J. C. (29 de junio de 2012). Eficiencia relativa en entidades ambientales gubernamentales. Recuperado el 15 de agosto de 2017, de: http://www.scielo.org.co/pdf/inun/v17n1/v17n1a12.pdf

CENTRO DE MONITORES DE DESPLAZAMIENTO INTERNO. (16 de enero de 2014). COLOMBIA: EI desplazamiento continua a pesar de esperanzas de paz. Recuperado el 20 de agosto de 2017, de: http://www.acnur.org/t3/uploads/media/2875 IDMC_Colombia_16ene2014.pdf

CERREJON. (2010). Mundo Cerrejón(61), 20.

CINEP. (2016). Minería, Conflinctos agrarios y ambientales en el sur de la guajira. Bogotá.

confetayrona.org. (16 de noviembre de 2015). Linea negra. Recuperado el 23 de agosto de 2017, de: http://www.confetayrona.org/images/pueblo-arhuaco/quienes-somos/que-queremos/quequeremos3.png

CONGRESO DE COLOMBIA. (4 de marzo de 1991). D.O. 39.720. Recuperado el 20 de agosto de 2017, de: http://www.alcaldiabogota.gov.co/sisjur/normas/Norma1.jsp?i=37032

CONTRALORÍA GENERAL DE LA REPÚBLICA. (2013). Fundamentos para superar el modelo estrativista. Bogotá. D.C: Imprenta nacional.

CONTRALORÍA GENERAL DE LA REPÚBLICA. (2013). Informe CGR mineria. Recuperado el 10 de septiembre de 2017, de:

https://www.google.com/url?q=http://www.contraloria.gov.co/documents/20181/472306/01 CGR mineria | 2013 comp.pdf/40d982e6-ceb7-4b2e-8cf25d46b5390dad\&sa=D\&ust=1505769078051000\&usg=AFQjCNHu5kLoKyr4xFANzh3PTxa6o6Cmm g

CORPOGUAJIRA. (20 de abril de 2010). Informe de Calidad de Aire Zona de inluencia Explotación Minera. Recuperado el 28 de agosto de 2017, de: http://corpoguajira.gov.co/wp/informecalidad-del-aire-zona-de-influencia-explotacion-minera-primer-trimestre-ano-2010/ 
CORTE CONSTITUCIONAL. (29 de octubre de 2009). MP. Nilson Pinilla Pinilla. Recuperado el 27 de agosto de 2017, de:

https://www.google.com/url?q=http://www.corteconstitucional.gov.co/relatoria/2009/t-76909.htm\&sa=D\&ust=1505769078053000\&usg=AFQjCNFpcVk2jct2-xS6-ZkzBkAMFVIJ6Q

CORTE CONSTITUCIONAL. (3 de febrero de 1997). MP. Antonio Barrera Carbonell. Recuperado el 27 de agosto de 2017, de: http://www.corteconstitucional.gov.co/relatoria/1997/su039-97.htm

CORTE CONSTITUCIONAL. (10 de noviembre de 1998). MP. Carlos Gaviria Díaz. Recuperado el 27 de agosto de 2017, de: http://www.corteconstitucional.gov.co/relatoria/1998/T-652-98.htm

CORTE CONSTITUCIONAL. (1 de julio de 2010). M. P. Garbriel Eduardo Mendoza Martelo. Recuperado el 1 de septiembre de 2017, de:

http://www.corteconstitucional.gov.co/relatoria/2010/t-547-10.htm

CORTE CONSTITUCIONAL. (14 de diciembre de 2010). MP. Nilson Pinilla Pinilla. Recuperado el 27 de agosto de 2017, de: http://www.corteconstitucional.gov.co/relatoria/2010/t-1045a-10.htm

CORTE CONSTITUCIONAL. (23 de septiembre de 2011). MP. Jorge Ignacio Pretelt Chaljub. Recuperado el 27 de agosto de 2017, de:

https://www.google.com/url?q=http://www.corteconstitucional.gov.co/RELATORIA/2011/T693-11.htm\&sa=D\&ust=1505769078036000\&usg=AFQjCNFNrbXXgely9iQ|Pitxpz5L29Xe-w

CORTE CONSTITUCIONAL. (3 de marzo de 2011). MP.Jorge Ivan Palacio Palacio. Recuperado el 27 de agosto de 2017, de: http://www.corteconstitucional.gov.co/relatoria/2011/t-129-11.htm

CORTE CONSTITUCIONAL. (14 de Septiembre de 2014). MP. Humberto Antonio Sierra Porto. Recuperado el 27 de agosto de 2017, de:

http://www.corteconstitucional.gov.co/relatoria/2010/t-745-10.htm

CORTE CONSTITUCIONAL. (5 de noviembre de 2014). MP. Martha Victoria Sáchica Méndez . Obtenido de http://www.corteconstitucional.gov.co/relatoria/2014/T-819-14.htm

CORTE CONSTITUCIONAL. (27 de abril de 2017). MP. Antonio José Lizarazo Ocampo. Recuperado el 12 de septiembre de 2017, de Auto 205 de 2017:

https://www.google.com/url?q=http://www.corteconstitucional.gov.co/relatoria/autos/2017/A 205-17.htm\&sa=D\&ust=1505769078044000\&usg=AFQjCNHIndqqiMnj7X1Ootv3XSn3sETkew 
DEFENSORÍA DEL PUEBLO. (8 de mayo de 2017). Defensor denuncia inconsistencias en el proceso de consulta con los pueblos indígenas en el mecanismo del Fast Track. Recuperado el 10 de agosto de 2017, de:

http://www.defensoria.gov.co/es/nube/noticias/6345/Defensor-denuncia-inconsistencias-enel-proceso-de-consulta-con-los-pueblos-ind\%C3\%ADgenas-en-el-mecanismo-del-Fast-TrackReuni\%C3\%B3n-ind\%C3\%ADgenas-Fast-Track-concertaci\%C3\%B3n-pueblos-consulta-previa-i

DRUMMONDCO. (s.f). Cronología. Recuperado el 3 de agosto de 2017, de: http://www.drummondco.com/acerca-de-nosotros/cronologia/?lang=es

El Heraldo. (29 de 1 de 2012). Minería llegal Contamina río en Sierra Nevada. Recuperado el 25 de Julio de 2017, de: https://www.elheraldo.co/region/mineria-ilegal-contamina-rio-en-sierra$\underline{\text { nevada-54959 }}$

ElEspectador.com. (6 de mayo de 2013). 'Colombia no está preparada para la locomotora minera'. Recuperado el 25 de agosto de 2017, de: http://www.elespectador.com/noticias/medioambiente/colombia-no-esta-preparada-locomotora-minera-articulo-420422

ElEspectador.com. (18 de agosto de 2017). Incineran otro centro ceremonioal indígena en la Sierra Nevada de Santa Marta. Recuperado el 5 de septiembre de 2017, de:

http://www.elespectador.com/noticias/medio-ambiente/incineran-otro-centro-ceremonialindigena-en-la-sierra-nevada-de-santa-marta-articulo-708880

ElEspectador.com. (18 de agosto de 2017). Queman centro Ceremoniales del pueblo Kankuamo en la Sierra Nevada de Santa Marta. Recuperado el 10 de septiembre de 2017, de: http://www.elespectador.com/noticias/medio-ambiente/queman-centros-ceremoniales-delpueblo-kankuamo-en-la-sierra-nevada-de-santa-marta-articulo-708731

ElHeraldo.com. (21 de enero de 2013). Cultivos de algodón se encuentran a punto de desaparecer en el Cesar. Recuperado el 2 de agosto de 2017, de:

https://www.elheraldo.co/noticias/economia/cultivos-de-algodon-se-encuentran-a-punto-dedesaparecer-en-el-cesar-97005

ElHeraldo.com. (2 de febrero de 2014). Minería: los costos ambientales de su operación. Recuperado el 1 de agosto de 2017, de: https://www.elheraldo.co/economia/mineria-los-costosambientales-de-su-operacion-141344 
ELHeraldo.com. (15 de febrero de 2016). Indigenas Suspenden Consulta previa. Recuperado el 2 de septiembre de 2017, de:

https://www.google.com/url?q=https://www.elheraldo.co/magdalena/indigenas-de-la-sierrasuspenden-los-procesos-de-consulta-previa-

243656\&sa=D\&ust=1505769078040000\&usg=AFQjCNHBxnO7m1FyWRyOknCfhax1 Yzw5A

EQUIPO ÁREA DE ACCIONES PÚBLICAS, Cajar. (1 de Mayo de 2015). Minería: ¿A Niños y a Niñas quién los Protege? Recuperado el 15 de Agosto de 2017, de:

https://www.colectivodeabogados.org/?Cerrejon-compro-terreno-para-desplazar-a-

comunidad-y-dejo-a-ninas-y-ninos-sin

EXTRACTIVISMO EN COLOMBIA. (8 de 10 de 2015). La Desviación del Río Rancheria ¿Un Crimen de Lesa Humanidad? Recuperado el 28 de julio de 2017, de:

http://extractivismoencolombia.org/la-desviacion-del-rio-rancheria-un-crimen-de-lesahumanidad/

FEDESARROLLO. (2008). Recuperado el 28 de julio de 2017, de:

http://www.repository.fedesarrollo.org.co/handle/11445/1012

FEDESARROLLO. (abril de 2008). Recuperado el 26 de julio de 2017, de:

http://www.repository.fedesarrollo.org.co/bitstream/handle/11445/1012/Repor_Abril \%20200

8 Cardenas y Reina.pdf?sequence $=3$

FEDESARROLLO. (2008). Cuadernos de Fedesarrollo. Recuperado el 10 de julio de 2017, de: http://www.repository.fedesarrollo.org.co/bitstream/handle/11445/893/CDF No 25 Abril 20 08.pdf? sequence $=1$

FIERRO Morales, J. (2012). Políticas Mineras en Colombia (Vol. 1). (J. Estrada Álvarez, Ed.) Bogotá: Instituto Latinoamericano para una Sociedad y un Derecho Alternativo.

GLENCORE. (s.f). Time line. Recuperado el 3 de agosto de 2017, de:

http://www.glencore.com/public-positions/supporting-development-in-colombia/prodecosoperations-in-colombia/

JIMENÉZ García, I. d. (2016). Aspectos Ambientales y de Comunidades que se Deben Tener en Cuenta en la Contratación y Titulación Minera: Diicultades y Retos (Vol. 1). Bogotá, Colombia: Universidad Externado. 
Marlon de Jesús Correa Fernández

LÓPEZ Montaño, C. (30 de abril de 2015). La enfermedad holandesa en la economía colombiana. Recuperado el 1 de agosto de 2017, de:

http://pensamiento.unal.edu.co/fileadmin/recursos/focos/focomineria/docs/cecilia lopez foro 30.04.2015.pdf

MINAMBIENTE. (s.f.). Recuperado el 1 de septiembre de 2017, de:

http://biblovirtual.minambiente.gov.co:3000/DOCS/MEMORIA/MMA-482/MMA-

482 CAPITULO03.pdf

ORGANIZACIÓN INTERNACIONAL DEL TRABAJO. (27 de junio de 1989). C169 - Convenio sobre pueblos indígenas y tribales, 1989. Obtenido de:

http://www.ilo.org/dyn/normlex/es/f?p=NORMLEXPUB:12100:0::NO::P12100 ILO CODE:C169

PEACE Brigades. (noviembre de 2011). Colombia: Minería en colomnbia a qué precio. Recuperado el 3 de agosto de 2017, de:

http://www.peacebrigades.org/fileadmin/user files/projects/colombia/files/colomPBla/111122 boletin final web.pdf

PORTAFOLIO. (10 de mayo de 2015). Recuperado el 15 de agosto de 2017, de: http://www.portafolio.co/negocios/empresas/regulador-toronto-castiga-pacific-coal-39710

PROCURADURÍA GENERAL DE LA NACIÓN. (2010). Informe preventivo. Bogotá.

SALINAS Alvarado, C. E. (27 de septiembre de 2011). Revista de Derecho del Externado Nueva Serie. Recuperado el 11 de septiembre de 2017, de La consulta previa como requisito obligatorio dentro de trámites administrativos cuyo contenido pueda afectar en forma directa a comunidades indígenas y tribales en Colombia:

http://revistas.uexternado.edu.co/index.php/derest/article/view/3019/3055

SAMUELSON, P. A., \& Nordhaus, W. D. (2010). Economía Con Aplicaciones a Latioamérica (19 ed., Vol. 1). (A. Deras Quiñones, Trad.) Ciudad de México D.F., México: McGrawHill.

Semana.com. (3 de septiembre de 2017). La preocupante radriografía de la Sierra Nevada por cuenta de la minería. Recuperado el 10 de septiembre de 2017, de:

http://sostenibilidad.semana.com/medio-ambiente/articulo/mineria-en-la-sierra-nevada-desanta-marta-indigenas-denuncian-afectaciones/38555 
Marlon de Jesús Correa Fernández

SHANTZ, P. (4 de Noviembre de 2011). colombia Analysis: Who is Paying Colombian Armed Groups for Acces to Gold? Recuperado el 10 de Agosto de 2017, de:

https://cpt.org//cptnet/2011/11/04/colombia-anyalysis-who-paying-colombian-armed-groupsaccess-gold

Sunat. (s.f). Recuperado el 24 de julio de 2017, de:

http://www.sunat.gob.pe/pdt/pdtModulos/independientes/regaliaMinera/

tradingeconomics.com. (5 de marzo de 2016). Historical. Recuperado el 30 de julio de 2017, de: https://tradingeconomics.com/commodity/coal

VICENTE, A., MARTIN, N., JAMES, D., BIRSS, M., LEFEBVRE, S., \& BAUER, B. (2011). Minería en Colombia: ¿A qué precio? Bogotá: CÓDICE LTDA.

VILLARAGA Sarmiento , Á. (2008). Cuando la madre tierra llora: crisis en derechos en humanos y humanitaria en la Sierra Nevada De Gonawindúa (SANTA MARTA). Bogotá D. C.: Fundación Cultura Democrática. 